\title{
Equidistant frequency triplets in pulsating stars: the combination mode hypothesis
}

\author{
M. Breger ${ }^{1}$ and K. Kolenberg ${ }^{1,2}$ \\ 1 Department of Astronomy, University of Vienna, Türkenschanzstrasse 17, 1180 Vienna, Austria \\ e-mail: michel.breger@univie.ac.at \\ 2 Institute of Astronomy, University of Louvain, Celestijnenlaan 200B, 3001 Heverlee, Belgium \\ e-mail: kolenberg@astro.univie.ac.at
}

Received 12 June 2006 / Accepted 15 August 2006

\begin{abstract}
Context. Multiplet structures are a common feature in pulsating stars, and can be the consequence of rotational splitting, mode interaction or sinusoidal amplitude variations.

Aims. In this paper we examine the phenomenon of (nearly) equidistant triplets, which are unlikely to be caused by rotational splitting, in different types of pulsating stars: a $\delta$ Scuti variable (1 Mon), an RR Lyrae variable (RR Lyr) and a short-period Cepheid (V473 Lyr). We examine the hypothesis that one of the modes forming the triplet results from a combination of the other two modes.

Methods. The analyses were carried out on recent data sets by using multiple-frequency analyses and statistics with the package PERIOD04. In particular, the small departures from equidistance were calculated for the three selected stars.

Results. For the $\delta$ Scuti variable 1 Mon, the departure from equidistance is only $0.000079 \pm 0.000001 \mathrm{~cd}^{-1}$ (or $0.91 \pm 0.01 \mathrm{nHz}$ ). For 1 Mon the Combination Mode Hypothesis with a mode excited by resonance is the most probable explanation. For the star RR Lyr, the hypothesis of resonance through a combination of modes should be considered. The results for the best-studied cepheid with a Blazhko period (V473 Lyr) are inconclusive because of an unfavorable period of 1.49d and insufficient data.
\end{abstract}

Key words. stars: oscillations $-\delta$ Sct - stars: variables: RR Lyr - Cepheids

\section{The problem}

The different frequencies excited in multimode stellar pulsators are an important tool to increase our understanding of stellar structure and evolution. Equidistant or near-equidistant frequency triplets form a special case, and are found in different types of pulsators. Here the frequency triplets have different physical origins which need to be examined carefully. Examples are white dwarfs (rotational splitting of nonradial modes: e.g., Winget et al. 1991), sdB stars (see Vučković et al. 2006 for a puzzling triplet) and roAp stars (oblique pulsator model). In this paper we examine a $\delta$ Scuti star (1 Mon: see Shobbrook \& Stobie 1974), a RR Lyrae star (RR Lyr: see Smith et al. 2003; Kolenberg et al. 2006), as well as a cepheid (V473 Lyr: equidistant spacing reported by Koen 2001).

Equidistant or nearly equidistant frequency triplets can be explained in several ways:

(i) Amplitude variability: amplitude variability gives rise to a number of separate peaks in a Fourier diagram. The number, height and separation of these peaks depend on the data coverage as well as the type of amplitude variability. A very special case exists: sinusoidal amplitude variability leads to an equidistantly spaced frequency triplet with two side peaks of equal height. This was originally proposed to explain the roAp stars (Kurtz 1982) through the Oblique Pulsator Model. In general, the peaks are not equidistant: to avoid the common spurious multiple peaks caused by amplitude variability and limited coverage, independent data samples need to be compared to each other.

(ii) Rotational splitting of nonradial modes: physical and geometric effects: rotational splitting of nonradial modes is a promising explanation for equidistant triplets only for stars that rotate extremely slowly (such as white dwarfs). Depending on which modes are examined, the rotational splitting may correspond to the rotation of the interior, which provides an important tool for asteroseismolgy. Second-order effects in rotational splitting become very important for modes dominant in stellar envelopes. In the presence of stellar rotation in excess of a few $\mathrm{km} \mathrm{s}^{-1}$, p-mode splitting will not be symmetric and equidistant triplets will not be seen (Pamyatnykh 2000). An example is the moderately rotating $\left(66 \pm 16 \mathrm{~km} \mathrm{~s}^{-1}\right) \delta$ Scuti star FG Vir: an $\ell=1$ triplet is observed to have separations of 0.42 and $0.64 \mathrm{c} / \mathrm{d}$ (Zima et al. 2006).

(iii) Successive radial orders: in the asymptotic pulsation limit, successive radial orders are equidistantly spaced. If three successive radial orders are excited, an equidistant frequency triplet can result. Such cases can be recognized easily because of the relatively large and predictable frequency differences between radial orders and will not be considered here.

(iv) The Combination Mode Hypothesis: this hypothesis, examined in the next section, requires an exact equidistant frequency triplet. In the case of resonance excitation, an almost equidistant frequency triplet also fits the hypothesis.

\section{Combination frequencies as an explanation for triplets}

The occurrence of linear combinations of frequencies is a common feature in pulsation phenomena. Such combinations often occur in the frequency spectra of stars pulsating with multiple 


\section{Combination Mode Hypothesis}

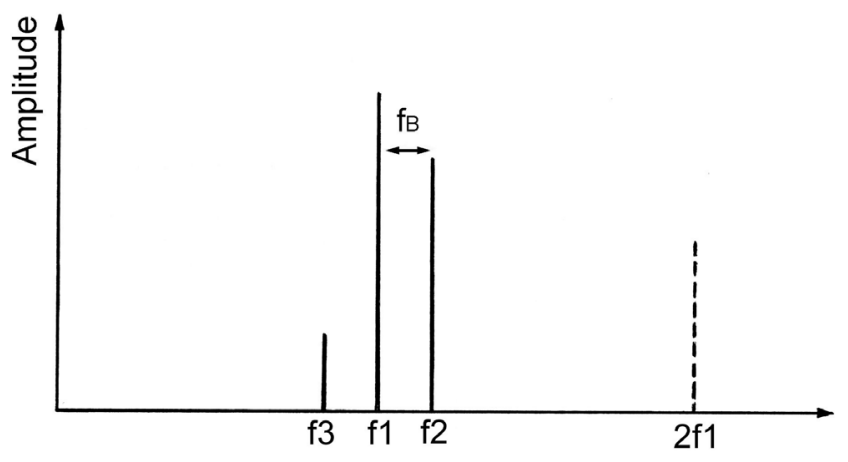

Frequency

Fig. 1. The triplet frequency $f_{3}$ results from the combination $2 f_{1}-f_{2}$. We examine the departure from equidistance, $\delta f=f_{2}+f_{3}-2 f_{1}$.

oscillation modes and are even seen in small-amplitude pulsators such as $\delta$ Scuti stars (Breger et al. 1999) and white dwarfs (Wu 2001).

In this paper we study the possibility of triplet structures resulting from mode combination and/or resonance phenomenon. The basic idea of the combination mode hypothesis is illustrated in Fig. 1. Assume that two modes $f_{1}$ and $f_{2}$ are excited and let $f_{1}$ have the highest amplitude. As a consequence, its harmonic frequency $2 f_{1}$ is also likely to occur in the frequency spectrum. Since a real second mode is excited at $f_{2}$, the combination $2 f_{1}-f_{2}$ may also occur. This combination is observed at $f_{3}$ and as a consequence a triplet is observed around $f_{1}$. The difference $f_{2}-f_{1}$ is referred to as $f_{B}$, the beat frequency (Fig. 1). It plays a crucial role in understanding RR Lyrae stars showing the Blazhko effect (see Sect. 4).

It is possible that the left-side or lower-frequency peak in the triplet, $f_{3}$, may not be located at the exact frequency of the combination. If there exists another mode in the pulsation spectrum of the star with an eigenfrequency close to that of the combination mode frequency, it may be excited through a resonance with the combination mode frequency and gain observable amplitudes. This additional mode has a frequency at or close to the combination frequency. Resonances are known to play important roles in determining the pulsational behavior of stars and we refer to an excellent theoretical discussion by Buchler et al. (1997).

Consequently, one should examine the departure from equidistance in the triplet:

$\delta f=\left(f_{2}-f_{1}\right)-\left(f_{1}-f_{3}\right)=f_{2}+f_{3}-2 f_{1}$.

If the triplet is not exactly equidistant, i.e., $\delta f \neq 0$, it is possible to exclude that the frequency is a pure combination frequency. This would strengthen the case of a resonance invoked by a combination frequency.

A further argument concerns the amplitude of the observed third frequency, which is either the combination frequency itself or a resonance excitation near the combination frequency. Regrettably, there are no one-to-one relationships between the amplitudes of the combination and the main frequencies involved in the combinations (Garrido \& Rodríguez 1996). Nonlinear pulsation favours the combination frequency terms $\left(i f_{1}+j f_{2}\right)$ where both $i$ and $j$ are positive (Antonello \& Aikawa 1998). Observational confirmation can be found for double mode cepheids (Pardo \& Poretti 1997), and in Delta Scuti stars (Table 4 of Breger et al. 2005). The difference in combination mode amplitude sizes even hold for a triple-mode AC And-type pulsator, V823 Cas (Jurcsik et al. 2006).

In case of resonance excitation it might be possible to see both the combination frequency and the mode excited by resonance at nearly the same frequency. Due to the closeness of the two frequencies, they might not be detected as two separate frequencies. Nevertheless, if both are present, the observer sees a single frequency with very long-term amplitude modulation with timescales of decades. Through phase locking, the resonant mode coupling may eventually result in a state of constant amplitude (e.g., as proposed for bump cepheids, Buchler et al. 1990).

In the case of an exact frequency match we have two possibilities: the combination mode hypothesis and resonant coupling leading to a steady limit cycle.

\section{Application to the $\delta$ Scuti variable 1 Mon}

\subsection{Available data and frequency analysis}

The $\delta$ Scuti variable 1 Mon is of special interest because it shows an almost equidistantly spaced frequency triplet with a separation of $0.1291 \mathrm{~cd}^{-1}(1.49 \mu \mathrm{Hz})$. It also has a low projected rotational velocity of $14 \mathrm{~km} \mathrm{~s}^{-1}$ (Balona et al. 2001).

An attractive interpretation of the frequency equidistance in terms of rotational splitting of a single nonradial mode fails, since the frequency triplet is composed of modes with different quantum numbers, $\ell$ (Balona $\&$ Stobie 1980; Balona et al. 2001). In particular, they have identified the dominant $7.35 \mathrm{~cd}^{-1}$ mode as a radial mode, which cannot show mode splitting. However, as Buchler et al. (1997) note, an observed multiplet of frequencies locked through a resonance is not necessarily associated with modes of the same degree $\ell$. A general condition for such coupling is given in Eq. (3.11) by Dziembowski (1982).

Furthermore, an explanation in terms of successive radial orders fails for several reasons, e.g., the frequency differences are too small by more than a factor of ten compared to predictions from the mean density of the star.

Nevertheless, the equidistance or near-equidistance should not be dismissed as an accidental agreement between three modes and provides an excellent opportunity to test the combination mode hypothesis. A key question is whether or not the frequencies are exactly equidistant.

To examine the nature of the frequency triplet, extensive observational studies with a long time base are needed. For the present analysis of 1 Mon, data from the following observing seasons were used: 1970/71 and 1971/72 (Millis 1973), 1972/73 (Shobbrook \& Stobie 1974), 1976/77 and 1977/78 (Balona \& Stobie 1980), 1978/79, 1982/83 and 1984/85 (Breger \& Fedotov 2006). The less extensive photometric data for the year 2000 (Balona et al. 2001) were not used since they are not publicly available. Hereafter, the data sets used are referred to as Mi, SS, $\mathrm{BS}$ and BF, respectively.

For the four data sets, we have analyzed the multiple frequencies using least-squares statistical methods (Period04, see Lenz \& Breger 2005). The zero-point shifts discussed in BF were applied to the Odessa data. Thirteen frequencies were found with the required significance of amplitude signal/noise ratio greater than 4.0 (Breger et al. 1993). These frequencies are the triplet, $f_{1}$ to $f_{3}$, discovered before, various combinations of these three frequencies and a new mode at $6.717 \mathrm{~cd}^{-1}$. The multifrequency analysis reveals additional peaks (e.g., at $9.544 \mathrm{~cd}^{-1}$ ), which could be additional pulsation modes. However, these peaks do not reach the adopted statistical significance level and are not listed here. Our results are shown in Table 1. 
Table 1. Multifrequency solution of 1 Mon.

\begin{tabular}{|c|c|c|c|c|c|c|}
\hline \multicolumn{2}{|c|}{ Frequency } & \multicolumn{5}{|c|}{$V$ amplitude (mag) } \\
\hline $\mathrm{cd}^{-1}$ & Name & & $1969 / 70$ to $1971 / 72$ & $1972 / 3$ & $1976 / 77$ to $1978 / 79$ & $1982 / 83$ to $1984 / 85$ \\
\hline & & Data: all & Mi & SS & $\mathrm{BS}, \mathrm{BF}$ & $\mathrm{BF}$ \\
\hline \multicolumn{7}{|c|}{ Main modes } \\
\hline 7.346153 & $f_{1}$ & 0.092 & 0.090 & 0.091 & 0.094 & 0.097 \\
\hline \pm 0.000002 & & & & & & \\
\hline 7.475269 & $f_{2}$ & 0.064 & 0.066 & 0.065 & 0.064 & 0.062 \\
\hline \pm 0.000005 & & & & & & \\
\hline 7.217116 & $f_{3}$ & 0.022 & 0.021 & 0.022 & 0.021 & 0.020 \\
\hline \multicolumn{7}{|l|}{ \pm 0.000005} \\
\hline \multicolumn{7}{|c|}{ Other frequencies } \\
\hline 6.717240 & $f_{4}$ & 0.004 & & & & \\
\hline 14.821422 & $f_{1}+f_{2}$ & 0.017 & & & & \\
\hline 14.692306 & $2 f_{1}$ & 0.014 & & & & \\
\hline 14.950538 & $2 f_{2}$ & 0.007 & & & & \\
\hline 14.563268 & $f_{1}+f_{3}$ & 0.005 & & & & \\
\hline 22.167575 & $2 f_{1}+f_{2}$ & 0.003 & & & & \\
\hline 22.296691 & $2 f_{2}+f_{1}$ & 0.003 & & & & \\
\hline 7.604385 & $2 f_{2}-f_{1}$ & 0.003 & & & & \\
\hline 22.038459 & $3 f_{1}$ & 0.003 & & & & \\
\hline 0.129116 & $f_{2}-f_{1}$ & $(0.002)$ & & & & \\
\hline
\end{tabular}

\subsection{Test of equidistance}

We can now calculate the departure from exact equidistance, $\delta f$, as defined by Eq. (1). This needs to be compared to the statistical uncertainties of the computed value, which depend on the accuracy of the three frequencies, $f_{1}, f_{2}$, and $f_{3}$. Our uncertainty values were calculated by carrying out extensive Monte Carlo simulations of the multifrequency sinusoidal fits. The results were checked by simple least-square error calculations which assume white noise and uncorrelated frequencies. The results of the two methods generally agreed to within $20 \%$ in the sense that the more realistic Monte Carlo simulations gave larger uncertainty values. The uncertainties are listed in Table 1.

From the four data sets covering 16 years we find

$\delta f=0.000079 \pm 0.000001 \mathrm{~cd}^{-1}$.

The high accuracy of $\delta f$ is not surprising if one considers the long time base and the resulting excellent frequency resolution. How much does the present result depend on the choice of data sets and our assumptions of constant amplitudes and periods? We have repeated our analysis by optimizing the frequencies allowing for variable amplitudes in the solution (see Table 1) or even variable amplitudes and frequencies. In both cases, the value of $\delta f=0.000079 \mathrm{~cd}^{-1}$ found earlier was obtained.

Slightly different values are computed if fewer data and a much shorter time base are used. If we choose only the Mi, SS and BS data (up to the $1977 / 78$ observing season), we find $\delta f=$ $0.000109 \pm 0.000004 \mathrm{~cd}^{-1}$. This compares well to the value of $\delta f=0.000115 \pm 0.000008 \mathrm{~cd}^{-1}$ reported for the same shorter data set by BS using a different multifrequency solution.

What happens if we assume equidistance? Not surprisingly, the combination mode, $2 f_{1}-f_{2}$, misses the third peak in the power spectrum completely with the new residuals still showing $f_{3}$. The solution finds $f_{3}$, but not at the predicted $2 f_{1}-f_{2}$ position.

The results indicate that the departure from equidistance is very small but statistically extremely significant. The departure from equidistance amounts to $0.9 \mathrm{nHz}$ or $6.8 \mathrm{~s}$ in a $3.3 \mathrm{~h}$ period. This also means that $f_{3}$ is not identical to the combination frequency $\left(2 f_{1}-f_{2}\right)$. Since the related combination $\left(2 f_{1}+f_{2}\right)$ has an observed amplitude of $0.003 \mathrm{mag}$, an amplitude of $0.022 \mathrm{mag}$ in the case of such an identification would have been difficult to explain. Consequently, the excitation by resonance explanation becomes very attractive: the combination frequency excites a nonradial mode (otherwise near stability) of almost the same frequency.

\section{Application to the RR Lyrae Blazhko star RR Lyr}

A considerable fraction of the RR Lyrae stars (20-30\% of the galactic RRab stars - fundamental radial mode pulsators - and about $5 \%$ of the RRc stars - first overtone radial mode pulsators) show a periodic amplitude and/or phase modulation, called the Blazhko effect. This phenomenon was discovered a century ago, and is still not well understood. The frequency spectra derived from externsive light and radial velocity curves of RR Lyrae Blazhko stars exhibit either a doublet structure or an equallyspaced triplet structure around the main pulsation frequency $f_{1}$ as well as its harmonics $k f_{1}$. The small frequency separation in the triplets/doublets corresponds to the Blazhko or modulation frequency $f_{B}$ (see Kovács 2001; Alcock et al. 2000, 2003). Since the main peak corresponds to a radial mode, rotational splitting can be ruled out. It is an important question as to why the multiplet structures occur with equal spacings at the harmonics just as at the main frequency.

\subsection{Resonance models and combination mode hypothesis for RR Lyrae stars}

The currently most cited models for explaining the Blazhko effect involve either a magnetic field or a resonance effect. In both types of models nonradial oscillation modes play a crucial role. The present resonance models suggest that the Blazhko effect is caused by a direct resonance between the main radial mode and a nonradial mode, most likely of a low degree $\ell$. Van Hoolst et al. (1998) showed that RR Lyrae stars have a very dense nonradial frequency spectrum, and that nonradial modes in the vicinity of the radial mode have a lower moment of inertia and hence are more prone to instability. Nowakowski \& Dziembowski (2001) investigated resonant excitation of nonradial modes in RR Lyrae 
stars and predicted significant amplitude (and phase) modulation in the case of excitation of a nonradial mode of low degree close to the radial mode. However, the strong nonlinear damping of nonradial modes at the amplitudes observed poses a major problem for such models (Nowakowsi \& Dziembowski 2003). Dziembowski \& Mizerski (2004) propose a scenario for the Blazhko effect, involving energy transfer from the radial to nonradial modes, but avoiding the problems of finite amplitude development uncovered by Nowakowski \& Dziembowski (2003).

For RR Lyrae stars a simple combination mode hypothesis was proposed by Borkowski (1981), who interpreted the numerous components in the light variation of AR Her as a nonlinear superposition of the main radial frequency $f_{1}$, and a mode at $2 f_{1}+f_{B}$. The additional mode was at first identified by Borkowski (1981) as the second or third radial overtone of the star. Later on, model calculations yielded the radial overtone modes at different frequencies and hence the additional mode must be a nonradial one (Kovács 1995). Recently, Kolenberg et al. (2006, hereafter K06) proposed a similar hypothesis to interpret the light curve variations of RR Lyrae as a combination of the main radial mode with a frequency $f_{1}$ and a nonradial mode at $f_{\mathrm{N}}=f_{1}+f_{B}$. These are the same frequencies as were considered by Balázs \& Detre (1943) to be at the basis of the amplitude modulation.

Alcock et al. (2000, 2003) examined the equidistance of triplet frequencies on a sample of RRc and RRab Blazhko stars found in the MACHO database. They defined the departure from equidistance as $f_{+}+f_{-}-2 f_{0}$, where $f_{0}, f_{-}$and $f_{+}$denote the main frequency (here $f_{1}$ ), the lower and the higher frequency side peak, respectively. For the RRc stars they found the deviations from equidistance to be insignificant (Alcock et al. 2000). For the RRab stars, the quantity $\delta f$ was, with few exceptions, within $\pm 0.0002 \mathrm{c} / \mathrm{d}$, half of the characteristic line width (Alcock et al. 2003). However, an analysis of artificial time series led to the conclusion that the observed small deviations from equidistant spacing cannot be accounted for by purely noise-induced frequency shifts. The authors state that the nonequidistant spacing has various origins, including cases in which neither the noise level nor peculiar additional features are suspected to be responsible for the deviation. Hence it is recommended to study the cause of non-equidistant frequency spacing on a star-by-star basis.

\subsection{Decomposing the RR Lyrae frequency spectrum}

We now examine the case of RR Lyr, the brightest Blazhko star, for which we used the photometric data published by K06. These data were obtained over a 421-day interval in 2003-2004. The data from only 2004 cover a 194-day interval, i.e., about 5 Blazhko cycles. RR Lyr is known to have a radial pulsation period $f_{1}=1.7642 \mathrm{c} / \mathrm{d}$. Its Blazhko or modulation period used to be about 40.8 days, but recent data show that it has decreased to about 39 days (K06).

For RR Lyrae Blazhko stars, usually a fit assuming intrinsically equidistant triplet frequencies is proposed, according to the following model:

$$
\begin{aligned}
f(t)= & A_{0}+\sum_{k=1}^{n}\left[A_{k} \sin \left(2 \pi k f_{1} t+\phi_{k}\right)\right. \\
& +A_{k+} \sin \left(2 \pi\left(k f_{1}+f_{B}\right) t+\phi_{k+}\right) \\
& \left.+A_{k-} \sin \left(2 \pi\left(k f_{1}-f_{B}\right) t+\phi_{k-}\right)\right] \\
& +B_{0} \sin \left(2 \pi\left(f_{B} t+\phi_{B}\right)\right)
\end{aligned}
$$

where $f_{1}$ is the main frequency and $f_{B}$ the Blazhko frequency. The results of the fit according to Eq. (2) were published by K06. They represent the common way of decomposing the frequency spectrum of a Blazhko star.

We now examine the recent RR Lyr data set published by K06 in terms of the equidistance of the triplet frequencies. We first consider the first order triplet, i.e., the triplet at the main frequency. The triplets at higher orders are considered in Sect. 4.4. As Alcock et al. (2003) concluded from their extensive study on the LMC fundamental mode RR Lyrae Blazhko stars, the higher (right-hand side) frequencies in the triplets have higher amplitudes in about $75 \%$ of the cases. RR Lyr also falls among the stars with right-side peaks higher than the left-side peaks.

\subsection{Equidistance at the first order triplet}

Instead of assuming a least-squares fit according to Eq. (2), we allow triplet structures which may not be exactly equidistant, leaving free the exact value of the side peak frequencies. Hence we construct a fit as follows:

$$
\begin{aligned}
f(t)= & A_{0}+\sum_{k=1}^{n}\left[A_{k} \sin \left(2 \pi k f_{1} t+\phi_{k}\right)\right. \\
& +A_{k+} \sin \left(2 \pi\left(f_{k+}\right) t+\phi_{k+}\right) \\
& \left.+A_{k-} \sin \left(2 \pi\left(f_{k-}\right) t+\phi_{k-}\right)\right] \\
& +B_{0} \sin \left(2 \pi\left(f_{B} t+\phi_{B}\right)\right)
\end{aligned}
$$

As for 1 Mon, the noise-induced frequency shifts were determined by means of Monte Carlo simulations with extensive numbers of iterations to obtain stability of the calculated values of the uncertainty. The departure from equidistance in the first order triplet is $\delta f=0.00072 \pm 0.00018 \mathrm{c} / \mathrm{d}$. This result calls for caution in the standard fitting of Blazhko stars with equidistant triplet structures.

\subsection{Extension to higher order triplets}

We now consider the departure from equidistance, $\delta f$, for the higher order triplets up to the fourth order, i.e., the components having significant amplitudes. Table 2 gives the departures from equidistance calculated in the standard way $\left(\delta f=f_{2}+f_{3}-2 f_{1}\right)$, Col. 4 in Table 2.

However, in the combination hypothesis for the higher order side peaks, it would be more appropriate to calculate the departure from the expected position of the combination frequency, $\delta f_{\mathrm{C}}$, rather than checking the departure from equidistance. For the first order triplet both terms yield the same value, but for the higher order triplets the values are different.

According to the combination hypothesis, the side peaks result from a sum (right side peaks) or difference (left side peaks) combination of the nonradial component $f_{\mathrm{N}}$ with $k f_{1}$ with $k=1,2, \ldots$ (up to the highest significant harmonic order). Hence, the departure from the expected position of the combination frequency should be calculated for each of the side peaks (left and right) separately. For example, in the combination hypothesis $f_{2-}=2 f_{1}-f_{B}$, the left side peak at twice the main frequency is equal to $3 f_{1}-f_{\mathrm{N}}$. The frequency is observed at $f_{2-}=3.50260 \pm 0.00012 \mathrm{c} / \mathrm{d}$. The frequency $3 f_{1}-f_{\mathrm{N}}$ is expected to occur at $3.502442 \pm 0.000028 \mathrm{c} / \mathrm{d}$. Hence the departure from equidistance $\delta f_{\mathrm{C}}$ is equal to $-0.00016 \pm 0.00012 \mathrm{c} / \mathrm{d}$. The departure from equidistance $\delta f$ yields a different value, $0.00015 \pm 0.00013 \mathrm{c} / \mathrm{d}$. A similar reasoning holds for the right side peaks in the triplets, and for all of the higher order triplet components. 
Table 2. Triplet freqencies, their uncertainties and the derived deviations based on the 2004 RR Lyr data published by Kolenberg et al. (2006). The departure from equidistance, $\delta f$, is given for the first four triplet orders. The last two columns give the departure from the expected position of the combination frequency for each of the side peak frequencies.

\begin{tabular}{cccccccc}
\hline \hline Triplet order & \multicolumn{3}{c}{ Triplet frequencies } & \multicolumn{3}{c}{ Deviation from: } \\
& & Central & Left & Right & Equidistancy & Expected combination \\
& $k$ & $f_{1}$ & $f_{2}$ & $f_{3}$ & $\delta f$ & Left & Right \\
\hline First order & 1 & 1.7641849 & 1.737533 & 1.790113 & 0.00072 & 0.00072 & - \\
& & \pm 0.0000022 & \pm 0.00018 & \pm 0.000028 & \pm 0.00018 & \pm 0.00018 & - \\
\hline Higher order & 2 & 3.5283698 & 3.50260 & 3.553953 & 0.00015 & -0.00016 & 0.00034 \\
& & \pm 0.0000044 & \pm 0.00012 & \pm 0.000049 & \pm 0.00013 & \pm 0.00012 & \pm 0.000049 \\
& 3 & 5.2925547 & 5.266189 & 5.318550 & -0.00037 & 0.00043 & -0.000067 \\
& & \pm 0.0000066 & \pm 0.00010 & \pm 0.000043 & \pm 0.00011 & \pm 0.00010 & \pm 0.000051 \\
& 4 & 7.0567396 & 7.030806 & 7.082288 & -0.00038 & 0.0000053 & 0.00038 \\
& \pm 0.0000088 & \pm 0.00016 & \pm 0.000049 & \pm 0.00017 & \pm 0.00016 & \pm 0.000057 \\
\hline
\end{tabular}

The combination hypothesis may be a plausible explanation for the triplet structures in RR Lyrae stars. Hence, it makes sense to consider an additional parameter for the higher order triplets, in addition to the previously defined one which is valid for the triplet around the main frequency, and which we apply in this paper to different types of pulsating stars.

As can be seen from Table 2, the departure from the expected position of the combination frequency for the higher order triplet structures is significant for some components, while for others it is not. The uncertainties given are derived from the uncertainties on the observed higher order side peak frequencies and the (first order) frequencies involved in the combination. They represent $1-\sigma$ uncertainties.

In general, we can conclude that the triplets in RR Lyrae Blazhko stars are not necessarily exactly equidistant, in accordance with the findings by Alcock et al. (2003). This should be considered in future studies of Blazhko stars. The departure from equidistance and/or from the expected position of the combination frequency may provide a test for the combination mode hypothesis to explain the typical frequency spectrum of a Blazhko star.

\section{The cepheid V473 Lyr}

V473 Lyr (HR 7308) is probably the best-studied cepheid with a strong Blazhko Effect. This $1.49 \mathrm{~d}$ cepheid has a modulation period near 1200d. Breger (1981) as well as Burki et al. (1982) have shown that a simple model based on beating between two closely spaced frequencies cannot explain the observed phase and amplitude variability. Furthermore, the long modulation period rules out rotational splitting of nonradial modes as well. An equidistant or near-equidistant frequency triplet has been reported for V473 Lyr: Koen (2001) analyzed the available Hipparcos photometry and proposed that the amplitude variations of V473 Lyr could be described by symmetrical frequency triplets separated by $0.0011 \mathrm{c} / \mathrm{d}$.

We have collected the available photometric data from 1966 to 1983 (Percy \& Evans 1980; Breger 1981; Henrikssen 1983; Burki et al. 1986; Breger 2006). We also included the excellent radial-velocity data by Burki et al. (1982). The periodicities in the data were analyzed with the PERIOD04 statistical package as in Sect. 2.

The multifrequency analysis confirmed the existence of the frequency triplet $\left(0.67075,0.67157\right.$, and $\left.0.66988 \mathrm{~cd}^{-1}\right)$. The frequency triplet misses equidistance by $0.00005 \pm 0.00001 \mathrm{~cd}^{-1}$. Because of the small formal error the result appears to be statistically significant. However, the solution may be less certain for the following reasons: (i) the fits are not good: the standard deviation is 0.023 mag per single measurement. (ii) Even after the inclusion of the radial-velocity data, the spectral window indicates that the frequencies and their harmonics are not statistically independent of each other. The unfavorable main pulsation period of $1.49 \mathrm{~d}$ represents a severe problem. (iii) Analysis of the residuals shows additional periodicities near the main frequencies.

We conclude that the data for the best-studied Blazhko cepheid are insufficient to test the combination mode hypothesis.

\section{Conclusion}

We have examined the phenomenon of (nearly) equidistant frequency triplets in a $\delta$ Scuti star, an RR Lyrae star, and a cepheid variable and tested whether the smallest amplitude component could be a combination of the other two modes. For the $\delta$ Scuti variable 1 Mon, a departure from equidistance of only $0.000079 \pm 0.000001 \mathrm{~cd}^{-1}$ (or $0.91 \pm 0.01 \mathrm{nHz}$ ). Here the Combination Mode Hypothesis with a mode excited by resonance is the most probable explanation. For the star RR Lyr, the hypothesis of resonance through a combination of modes was also found to be plausible. Finally, the results for the best-studied cepheid with a Blazhko period (V473 Lyr) were inconclusive because of an unfavorable period of $1.49 \mathrm{~d}$ and insufficient data.

Acknowledgements. Part of the investigation has been supported by the Austrian Fonds zur Förderung der wissenschaftlichen Forschung.

\section{References}

Alcock, C., Allsman, R., Alves, D. R., et al. 2000, ApJ, 542, 257

Alcock, C., Alves, D. R., Becker, A., et al. 2003, ApJ, 589, 597

Antonello, E., \& Aikawa, T. 1998, A\&A, 337, 39

Balázs, J., \& Detre, L. 1943, Communications of the Konkoly Observatory, No. 18,125

Balona, L. A., \& Stobie, R. S. 1980, MNRAS, 190, 931

Balona, L. A., Bartlett, B., Caldwell, J. A. R., et al. 2001, MNRAS, 321, 239

Blazhko, S. N. 1907, Astron. Nachr., 173, 325

Borkowski, K. J. 1980, Acta Astron., 30, 393

Breger, M. 1981, ApJ, 249, 666

Breger, M. 2006, CoAst, 148, in press

Breger, M., \& Fedotov, Yu. T. 2006, CoAst, 148, in press

Breger, M., Stich, J., Garrido, R. et al. 1993, A\&A, 271, 482

Breger, M., Handler, G., Garrido, R., et al. 1999, A\&A, 349, 225

Breger, M., Lenz, P., Antoci, V., et al. 2005, A\&A, 435, 955

Buchler, J. R., Moskalik, P., \& Kovács, G. 1990, ApJ, 351, 617

Buchler, J. R., Goupil, M.-J., \& Hansen, C. J. 1997, A\&A, 321, 159

Burki G., Mayor M., \& Benz W. 1982, A\&A, 109, 258

Burki, G., Schmidt, E. G., Arellano Ferro, A., et al. 1986, A\&A, 168, 139 
Dziembowski, W. A. 1982, Acta Astron., 32, 147

Dziembowski, W. A., \& Mizerski, T. 2004, Acta Astron., 54, 363

Garrido, R., \& Rodriguez, E. 1996, MNRAS, 281, 696

Henrikssen, G. 1983, Uppsala Astr. Obs. Report, 26

Jurcsik, J., Szeidl, B., Varadi, M., et al. 2006, A\&A, 445, 617

Koen, C. 2001, MNRAS, 322, 97

Kolenberg, K., Smith, H. A., Gazeas, K., et al. 2006, A\&A, in press

Kovács, G. 1995, A\&A, 295, 693

Kovács, G. 2001, in Stellar pulsation - nonlinear studies, ed. M. Takeuti, \& D. D. Sasselov, Astrophysics and Space Science Library, 257 (Dordrecht: Kluwer Academic Publishers), 61

Kurtz, D. W. 1982, MNRAS, 200, 807

Lenz, P., \& Breger, M. 2005, CoAst, 146, 53
Millis, R. L. 1973, PASP, 85, 410

Nowakowski, R. M., \& Dziembowski, W. A. 2001, Acta Astron., 51, 5 Nowakowski, R. M., \& Dziembowski, W. A. 2003, Ap\&SS 284, 273

Pamyatnykh, A. A. 2000, ASPC, 210, 215

Pardo, I., \& Poretti, E. 1997, A\&A, 324, 121

Percy, J. R., \& Evans, N. R. 1980, AJ, 85, 1509

Smith, H. A., Church, J. A., Fournier, J., et al. 2003, PASP, 115, 43

Shobbrook, R. R., \& Stobie, R. S. 1974, MNRAS, 169, 643

Van Hoolst, T., Dziembowski, W. A., \& Kawaler, S. D. 1998, MNRAS, 297, 536

Vučković, M., Kawaler, S. D., O'Toole, S., et al. 2006, ApJ, 646, 1230

Winget, D. E., Nather, R. E., Clemens, J. C., et al. 1991, ApJ, 378, 326

Wu, Y. 2001, MNRAS, 323, 248

Zima, W., Wright, D., Bentley, J., et al. 2006, A\&A, 455, 235 